\title{
MicroRNA-145 inhibits cell proliferation by directly targeting ADAM17 in hepatocellular carcinoma
}

\author{
YUWU LIU ${ }^{1-3}$, CHANG WU ${ }^{4}$, YING WANG ${ }^{1,2}$, SAILAN WEN $^{1,2}$, JUNPU WANG $^{1,2}$, \\ ZHIHONG CHEN ${ }^{1,2}$, QIONGQIONG HE ${ }^{1,2}$ and DEYUN FENG ${ }^{1,2}$
}

\author{
${ }^{1}$ Department of Pathology, Xiangya Hospital, Central South University, Changsha, Hunan 410008; \\ ${ }^{2}$ Department of Pathology, School of Basic Medical Sciences, Central South University, Changsha, Hunan 410013; \\ ${ }^{3}$ Department of Morphology, The Institute of Advanced Occupation Technology, Xinjiang Medical University, \\ Urumqi, Xinjiang 830011; ${ }^{4}$ Department of Pathology, Shenzhen Sixth People's Hospital (Nanshan Hospital), \\ Shenzhen, Guangdong 518052, P.R. China
}

Received April 13, 2014; Accepted July 25, 2014

DOI: $10.3892 /$ or.2014.3424

\begin{abstract}
MicroRNAs (miRNAs) are key regulators in cell processes. Emerging evidence has suggested that there is a direct association between miRNAs and cancer. However, the exact regulatory mechanisms of miRNAs in tumorigenesis are poorly understood. In the present study, we showed that miR-145 is able to significantly reduce mRNA and protein expression levels of A disintegrin and metalloproteinase 17 (ADAM17) in liver cancer cells (SMMC-7721, BEL-7402 and Huh-7). Dual luciferase reporter assays confirmed that ADAM17 is a direct target of miR-145. Notably, we found that miR-145 inhibits cell proliferation and growth activity in SMMC-7721 cells. These results demonstrated that it may be exert the function of tumor suppression in a particular link of cancer cell growth. Further studies revealed that the silencing of ADAM17 decreased the proliferation and growth activity of SMMC-7721 cells. Moreover, it reduced the expression of MMP-9. In conclusion, miR-145 inhibits liver cancer cell proliferation by directly targeting ADAM17. Thus, it may become a promising biological target in the treatment strategy of hepatocellular carcinoma.
\end{abstract}

\section{Introduction}

MicroRNAs (miRNAs) are a class of small cellular RNAs of 18-25 nucleotides in length that can play important regulatory roles in many cell events, including tumorigenesis and organ development (1-3). The biogenesis pathway of miRNAs contain more than one process, and there are six steps from miRNA genes to mature miRNA in miRISC,

Correspondence to: Dr Deyun Feng, Department of Pathology, Xiangya Hospital, Central South University, 87 Xiangya Road, Changsha, Hunan 410008, P.R. China

E-mail: deyunfeng2014@126.com

Key words: miR-145, ADAM17, MMP-9, hepatocellular carcinoma including transcription, cropping, export, dicing, strand selection and miRISC assembly. A variety of enzyme proteins are involved in these biological processes, such as RNA polymerase II (Pol II) and Drosha-DGCR8. Exp5-RanGTP exhibited a biological reaction within the nucleus, whereas Dicer-TRBP and Dicer-TRBP-Ago achieved their function in the cytoplasm (4). miRNAs can direct RISC to downregulate gene mRNA expression by mRNA cleavage or translational repression (1). Various calculation methods have been used to predict the targeted genes and target sites of miRNA, and it is estimated that over one third of human genes appear to be conserved miRNA targets (5).

The role of miRNA has been increasingly emphasized in the development and progression of liver disease. Results of previous studies have shown that miRNAs were associated with numerous types of liver disease, including non-alcoholic fatty liver disease, viral hepatitis and hepatocellular carcinoma (HCC) (6). HCC is the most common primary malignancy of the liver (7). The abnormal expression of miRNA in HCC has been previously identified and verified, with miR-21, miR-135a, miR-146a, miR-151, miR-221, miR-222, miR-192, miR-801, miR-194 and miR-618 being upregulated, and let-7g, miR-22, miR-26a, miR-27a, miR-29c, miR-122, miR-320, miR-491, miR-650 and miR-145 being downregulated in HCC. Additionally, the disorder of miRNA expression occurs in HCC associated with HBV or HCV $(6,8)$. A lower expression of miR-145 is also associated with many other tumors, including breast, colon, prostate, lung, bladder, pituitary, B-cell malignancies and ovarian. In addition, miR-145 is known to be important in the development of tumor, including cell growth, invasion and metastasis (9). However, the underlying mechanism involved remains to be determined.

A previous study demonstrated that some miRNAs may function as oncogenes or tumor suppressors (10). miR-145 is usually considered to be a tumor-suppressor gene. It can inhibit the occurrence and development of cancer by regulating oncogenes and/or genes that control cell growth, differentiation or apoptosis (11-13). A handful of target genes of miR-145 have been identified and validated, including YES and STAT1 (14), mucin 1 (13), HDAC2 (15), EGFR and NUDT1 (16), and 
CTGF (17). It has been found that miR-145 can repress the migration and invasion of tumor cells by targeting A disintegrin and metalloproteinase 17 (ADAM17) in several malignant tumors (18-20). ADAM17, also known as TNF- $\alpha$-converting enzyme (TACE), plays a vital role during the ectodomain shedding of TNF- $\alpha(21,22)$, and contributes to the progression of breast cancer and glioma $(23,24)$. TNF- $\alpha$ induces the $\mathrm{NF}-\kappa \mathrm{B}$ signaling pathway to mediate the matrix metalloproteinase-9 (MMP-9) expression $(25,26)$. Findings of a recent study showed that ADAM17 can mediate MMP-9 expression in lung epithelial cells (27). Therefore, we hypothesized that miR-145 is able to inhibit the HCC cell growth by moderating the ADAM17/MMP-9 pathway. In the present study, we aimed to identify the association between this pathway and hepatocarcinogenesis and determine whether miR-145 is a promising biomarker in the diagnosis and treatment of HCC.

\section{Materials and methods}

Cell culture. SMMC-7721, BEL-7402, Huh-7 and HEK-293T cells were purchased from the Cell Bank of the Chinese Academy of Sciences. SMMC-7721 and BEL-7402 cells were cultured in RPMI-1640 medium, supplemented with $10 \%$ (v/v) fetal bovine serum (FBS), while Huh-7 and HEK-293T cells were maintained in DMEM high-glucose medium supplemented with $10 \%$ (v/v) FBS (all from Gibco, Invitrogen Life Technologies, Grand Island, NY, USA). The media contained $100 \mathrm{U} / \mathrm{ml}$ penicillin and $100 \mu \mathrm{g} / \mathrm{ml}$ streptomycin. The cells were incubated at $37^{\circ} \mathrm{C}$ and supplemented with $5 \% \mathrm{CO}_{2}$ in the humidified chamber.

RNA oligonucleotides and transfection. The pre-miR $\mathrm{R}^{\mathrm{TM}}$ miR-145 precursors (miR-145) and pre-miR ${ }^{\mathrm{TM}}$ miRNA precursor negative control (NC) were obtained from Ambion, Inc. (Austin, TX, USA). Cell transfection was performed using siPORT $^{\text {тм }}$ NeoFX $^{\text {тм }}$ Transfection Agent (Ambion) according to the manufacturer's instructions.

Plasmid construction and transfection. The human ADAM17 mRNA sequence (NM_003183.4) was used to design siRNA target sequences according to the design principle of shRNA (28). Four siRNA target sequences (971-991, 1658-1678, 1864-1884 and 2540-2560) were selected. A negative control (shNC) siRNA sequence was used as a control for ADAM17 siRNA (Table I). CACC was added at the end of the sense strand template, which is complementary with the formed sticky end after BbsI enzyme digestion. GATC was added at the end of the antisense strand template, which is complementary with the formed sticky end after BamHI enzyme digestion. A stem-loop-stem conformation was selected to avoid the formation of termination signal. The structure of T6 was selected as the shRNA transcription termination sequence. DNA oligonucleotides chemically were purchased by the Shanghai GenePharma Co., Ltd. (Shanghai, China). The shRNAannealed oligonucleotides were ligated into pGPU6/GFP/Neo siRNA expression vectors (GenePharma) between the $B b s \mathrm{I}$ and BamHI sites by T4 DNA ligase (Takara Bio Inc., Shiga, Japan). The orientation of the inserted shRNA cassettes was verified by restriction enzyme analysis and DNA sequencing. Cell transfection was performed using Lipofectamine 2000
Table I. Target sequences for RNA interference in ADAM17 gene.

\begin{tabular}{lc} 
shRNA vector & Target sequence \\
\hline 971-ADAM17 & GGAACACTTCATGGGATAATG \\
1658-ADAM17 & GCATCATGTATCTGAACAACG \\
1864-ADAM17 & GCTGAAGATGACACTGTTTGC \\
$2540-A D A M 17$ & GCACAGCTGCCAAGTCATTTG \\
Negative control & GTTCTCCGAACGTGTCACGT \\
\hline
\end{tabular}

ADAM17, A disintegrin and metalloproteinase 17.

transfection reagent (Invitrogen, Carlsbad, CA, USA) according to the manufacturer's instructions.

Quantitative PCR and semi-quantitative RT-PCR. Total RNA was extracted from cultured cells using TRIzol (Invitrogen). Quantitative PCR (qPCR) was carried out to quantify the expression of miR-145. The TaqMan MicroRNA Transcription kit, TaqMan Universal Master Mix II and TaqMan assays were used according to the manufacturer's instructions (Invitrogen Life Technologies). U6 snRNA was used as the normalization control. Each sample was tested in triplicate and the relative gene expression was determined. The data were calculated using the $2^{-\Delta \Delta \mathrm{Ct}}$ method.

Semi-quantitative RT-PCR was performed to detect the expression of ADAM17. Total RNA ( $2 \mu \mathrm{g}$ ) was used for synthesis of Oligo(dT)-primed single-stranded cDNA using a First Strand cDNA Synthesis kit (Fermentas, Burlington, Canada). cDNA products were then amplified using Platinum PCR SuperMix, with GAPDH serving as an internal control for the total cDNA content. The oligonucleotide sequences used as PCR primers were: ADAM17 (forward), 5'-ATGACA TCTATCGGAACAC-3', and (reverse), 5'-GCTATAATAAGC CTTTGGAC-3'; GAPDH (forward), 5'-AATCCCATCACC ATCTTCC-3' and (reverse),5'-CATCACGCCACAGTTTCC-3'. PCR products were visualized by electrophoresis on ethidium bromide-stained $1.5 \%$ agarose gels, and the intensities of the bands were measured with the aid of Bio-Rad Image Analysis software. ADAM17 expression was evaluated by determining the ADAM17 mRNA/GAPDH mRNA ratio. Experiments were repeated at least three times.

Western blot analysis. The cells were treated in lysis buffer (50 mM Tris- $\mathrm{HCl}, 0.5 \%$ sodium deoxycholate, $25 \mathrm{mM}$ EDTA) containing protease inhibitor cocktail (Roche Diagnostics $\mathrm{GmbH}$ Mannheim, Germany) for $30 \mathrm{~min}$ on ice. Equal amounts of protein were separated by $10 \%$ SDS-PAGE gels, and transferred onto polyvinylidene difluoride membranes. After blocking with 5\% skim milk solution for $2 \mathrm{~h}$, the membrane was incubated with antibodies against ADAM17 (Abcam, Cambridge, UK) or MMP-9 (Cell Signaling, Danvers, MA, USA) or $\beta$-actin (Proteintech, Chicago, IL, USA) at $4^{\circ} \mathrm{C}$ overnight. The appropriate horseradish-conjugated secondary antibody was incubated for $1 \mathrm{~h}$ at room temperature, the protein signal was then identified using the chemiluminescence method (ECL; Auragene Bioscience Corporation, Hunan, China). 
Band intensities were quantified using Bio-Rad image analysis software. Experiments were repeated at least three times.

Luciferase activity assay. A 500-bp sequence containing the predicted miR-145 binding site at the 3'UTRs of ADAM17 or a 500-bp sequence containing a scrambled sequence was cloned into the XhoI/NotI site of the psiCHECK-2 vector (Promega, Madison, WI, USA) to generate PsiCHECK-2-ADAM17-WT and PsiCHECK-2-ADAM17-mut vectors, respectively. SMMC-7721 and HEK-293T cells were cultured in 24-well plates, and co-transfected with PsiCHECK-2-ADAM17-WT and PsiCHECK-2-ADAM17-mut, and miR-145 precursor or the negative control using Lipofectamine 2000 (Invitrogen). Luciferase assays were performed using a luciferase assay kit (Promega) according to the manufacturer's instructions. Firefly luciferase was used for normalization. Experiments were repeated at least three times.

Cell proliferation assay. SMMC-7721 cells $\left(4 \times 10^{3}\right.$ cells/well) were plated in 96-well plates and incubated for $24 \mathrm{~h}$. An MTT assay was performed to measure cell viability at 24, 48 and $72 \mathrm{~h}$ after transfection. The absorbance at $490 \mathrm{~nm}$ was measured using a microplate reader (Bio-Tek Instruments, Winooski, VT, USA). Each experiment was performed in six replicate wells and independently repeated three times.

Colony formation assay. SMMC7721 cells were collected at $48 \mathrm{~h}$ after transfection, counted and seeded in 6-well plates at $4 \times 10^{2}$ cells/well. During colony growth, fresh culture medium was replaced every 2-3 days and then cells were cultured for 2 weeks. The cells were washed twice in phosphate-buffered saline (PBS) and fixed by methanol for $15 \mathrm{~min}$. The fixed cells were stained with appropriate Giemsa solution for $30 \mathrm{~min}$. The cells were subsequently washed slowly in running water and air-dried. Colonies were counted only when they contained $>50$ cells. The ability of colony formation was assessed by the colony formation rate, calculated as the number of colonies/ number of seeded cells x $100 \%$. Experiments were repeated at least three times.

Cell cycle analysis. SMMC7721 cells were trypsinized at $48 \mathrm{~h}$ after transfection, and washed in PBS, fixed with $500 \mu \mathrm{l}$ of $70 \%$ ethanol at $-20^{\circ} \mathrm{C}$ overnight. Subsequently, the cells were stained with propidium iodide (PI) solution at $37^{\circ} \mathrm{C}$ for $30 \mathrm{~min}$. Analysis was performed on a FACS flow cytometer (BD Biosciences, San Jose, CA, USA). Experiments were repeated at least three times.

Cell invasion and migration assay. A transwell chamber (8- $\mu \mathrm{M}$ pore diameter; Corning Costar) was used to perform the assay. The filter was coated with Matrigel (BD Biosciences) for examination of cell invasion, while the filter without coated Matrigel was used for the migration assay. The transfected cells were resuspended in serum-free medium and $200 \mu 1$ cell resuspension solution (cell invasion, $1 \times 10^{5}$ cells/well; cell migration, $1 \times 10^{6}$ cells/well) was added to the hydrated Transwell chambers. Simultaneously, $500 \mu 1$ complete medium with $10 \%$ FBS was added to the lower chambers. The cells were cultured in the incubator for $12 \mathrm{~h}$ (cell migration) or $24 \mathrm{~h}$ (cell invasion). Subsequently, the cells on the upper surface of the membrane were removed with cotton swab. Filters were fixed in $4 \%$ formaldehyde for $30 \mathrm{~min}$ and stained with $0.1 \%$ crystal violet for $20 \mathrm{~min}$. The cells were counted under a microscope. Experiments were repeated at least three times.

Statistical analysis. Data are presented as the mean and standard error (means \pm SEM). The two-tailed Student's t-test, one-way ANOVA, Mann-Whitney U or Kruskal-Wallis tests were used for data analysis. The statistical analyses were carried out using SPSS 19.0 for windows. $\mathrm{P}<0.05$ was considered to indicate a statistically significant result.

\section{Results}

ADAM17 was directly regulated by miR-145 in HCC. The identification of an appropriate target gene for miRNA using miRNA-target gene prediction software is important in understanding its function. ADAM17 was predicted by TargetScan (5), PicTar (29) and miRanda (30) (Fig. 1A), and assayed as a target of miR-145. Expression levels of miR-145 were determined by qPCR in the SMMC-7721, BEL-7402 and Huh-7 cell lines (Fig. 1B), and found to be significantly lower in the SMMC-7721 and BEL-7402 cell lines as compared to the Huh-7 cell lines ( $\mathrm{P}<0.05$, Kruskal-Wallis test). miR-145 precursor and a negative control RNA were used to transfect the HCC cell line with SMMC7721, BEL-7402 and Huh-7 (Fig. 1C-E). Following transfection, miR-145 was significantly overexpressed in the three cell lines $(\mathrm{P}<0.01$, the two-tailed Student's t-test).

The expression of ADAM17 was detected by RT-PCR and western blotting, respectively. ADAM17 mRNA expression was decreased significantly in BEL-7402 and Huh-7 cells, however, it did not exhibit any change in SMMC-7721 cells (Fig. 2A). ADAM17 protein expression was markedly decreased in SMMC-7721 and Huh-7 cells, however, no change was observed in the BEL-7402 cells (Fig. 2B). To determine whether miR-145 directly regulates ADAM17 expression, a luciferase activity assay was performed. miR-145 overexpression reduced the activity of the luciferase reporter gene fused to the wild-type ADAM17 3'UTR in SMMC-7721 ( $\mathrm{P}<0.01$, Kruskal-Wallis test) and HEK-293T cells ( $\mathrm{P}<0.05$, one-way ANOVA) (Fig. 2C). These resutlts showed that miR-145 targets ADAM17 directly.

Effect of miR-145 on the cell proliferation, colony formation and cell cycle in HCC cells. To investigate whether the ectopic expression of miR-145 inhibits the viability and growth of cells in liver cancer tumorigenesis, miR-145 precursor or the negative control was transfected into SMMC-7721 cells. The MTT assay showed that the overexpression of miR-145 resulted in a significantly decreased cell proliferation at $24 \mathrm{~h}$ $(\mathrm{P}<0.01$, the two-tailed Student's t-test $), 48 \mathrm{~h}(\mathrm{P}<0.01$, the two-tailed Student's t-test) and $72 \mathrm{~h}(\mathrm{P}<0.01$, Mann-Whitney U test) (Fig. 3A). The colony-forming efficiency of SMMC-7721 cells transfected with miR-145 precursor was markedly lower than that of the control group $(\mathrm{P}<0.05$, Mann-Whitney $\mathrm{U}$ test) (Fig. 3B). These results demonstrated that miR-145 overexpression is able to suppress cell growth in HCC. Distribution of the cell cycle was determined by flow cytometry. The results showed that miR-145 overexpression resulted in a 


\section{A 3 ' ucCCUAAGGAC-CCUUUUGACCUg 5 ' hsa-miR-145 ||$|:||||||||||| \mid$
$5^{\prime}$ atttATTTGTGATGACAACTGGAa $3 '$ ADAM17}

B

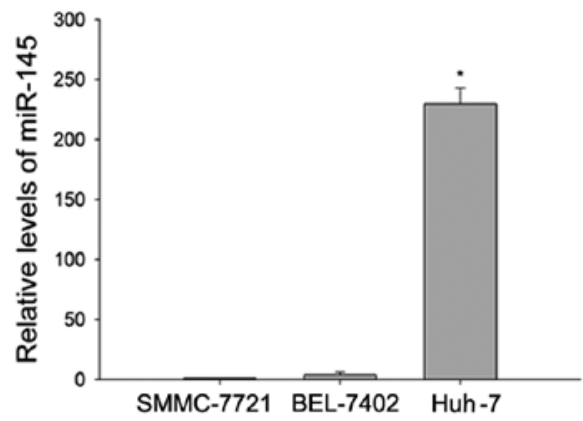

D



C

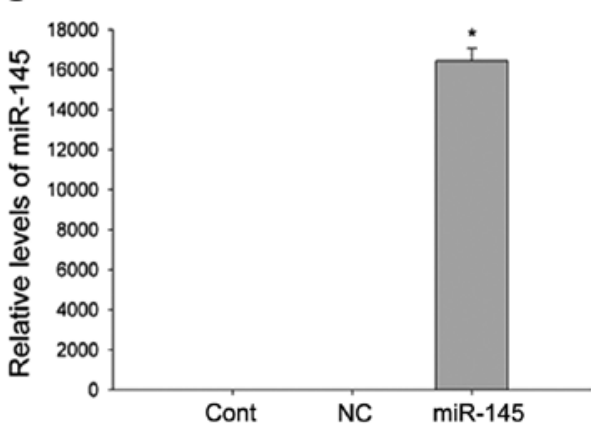

$\mathrm{E}$

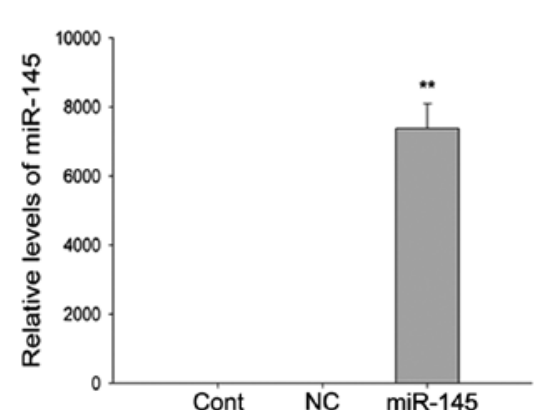

Figure 1. The sequence alignment of miR-145 binding site to the 3'UTR region of ADAM17 and the expression of miR-145 in HCC cell lines were shown. (A) The sequence alignment of miR-145 binding site to the 3'UTR region of ADAM17 is predicted by miRanda (V1.9). (B) The relative levels of miR-145 expression in HCC cell lines (SMMC-7721, BEL-7402 and Huh-7) were analyzed by qPCR. (C-E) The expression of miR-145 is significantly upregulated in liver cancer cells (SSMC-7721, BEL-7402 and Huh-7) transfected with miR-145 precursor as compared to the negative control (NC). ${ }^{*} \mathrm{P}<0.05 ;{ }^{* *} \mathrm{P}<0.01$. ADAM17, A disintegrin and metalloproteinase 17; HCC, hepatocellular carcinoma.

A

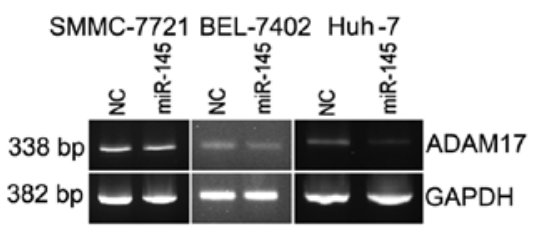

C

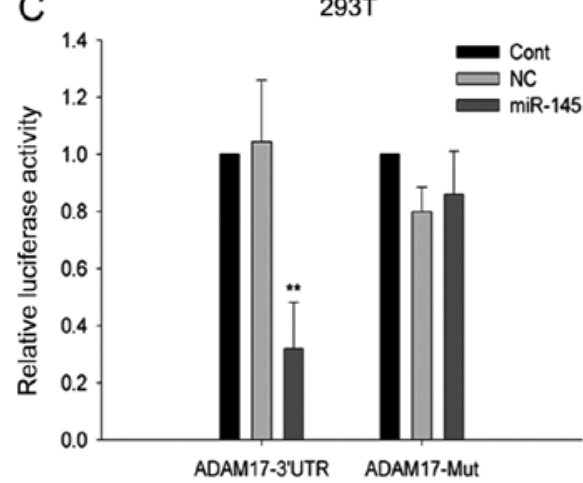

B $\quad$ SMMC-7721

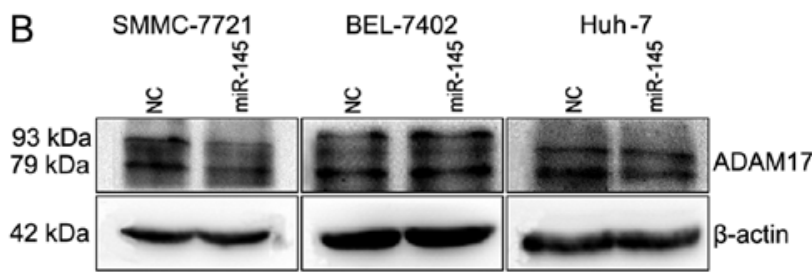

SMMC-7721

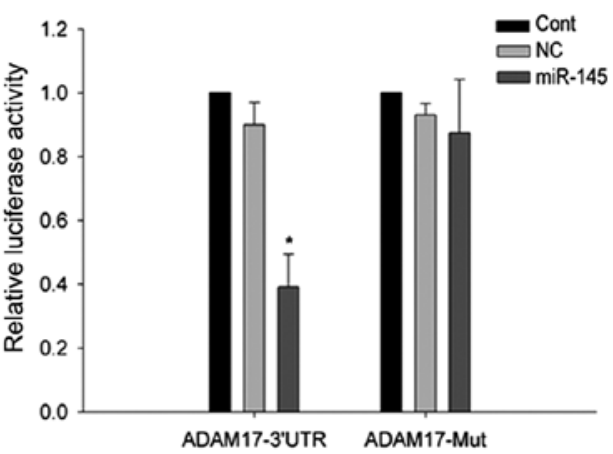

Figure 2. miR-145 suppresses ADAM17 by targeting its 3'UTR directly. (A and B) SMMC-7721, BEL-7402 and Huh-7 cells were transfected with miR-145 precursor (mir-145) and the negative control (NC), at $48 \mathrm{~h}$ after transfection. The mRNA and protein expression of ADAM17 was analyzed by RT-PCR and western blotting, as compared to NC. ADAM17 protein has two subunits with a molecular weight of 93 and $79 \mathrm{kDa}$, respectively. (C) Luciferase activity of wild-type or mutant ADAM17 3'UTR reporter gene in HEK-293T and SMMC-7721 cells transfected with miR-145 precursor or NC was determined. The results show that the luciferase activity of wild-type ADAM17 3'UTR transfected with miR-145 precursor was decreased as compared to other groups in the two cells. $\left({ }^{*} \mathrm{P}<0.05,{ }^{* *} \mathrm{P}<0.01\right)$. ADAM17, A disintegrin and metalloproteinase 17. 

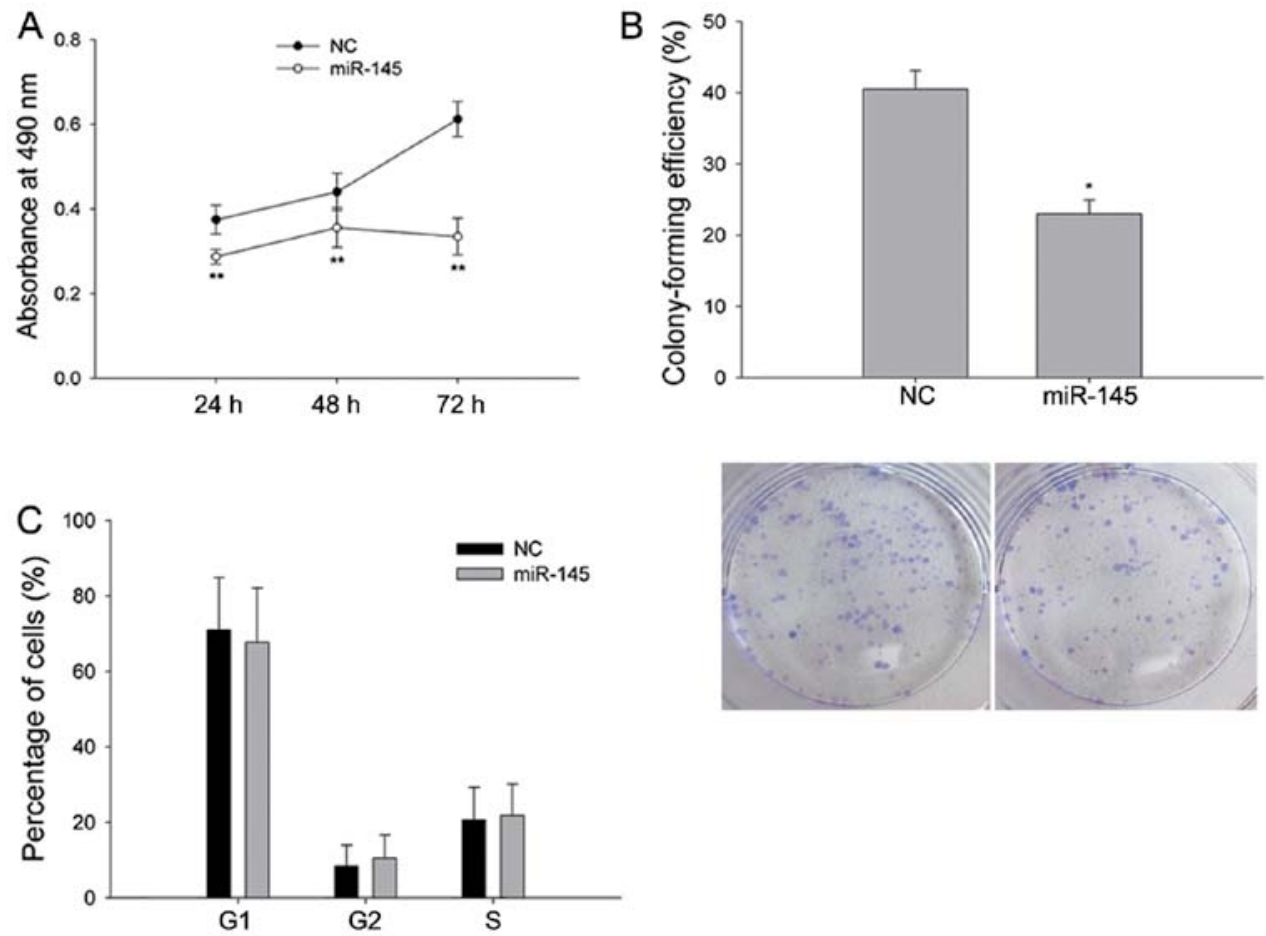

Figure 3. miR-145 overexpression reduces proliferation and colony formation ability of SMMC-7721 cells. (A) Viability of SMMC-7721 cells transfected with negative control RNA (NC) or miR-145 precursor (miR-145) was detected by the MTT assay ( $\left.{ }^{* *} \mathrm{P}<0.01\right)$. (B) SMMC-7721 cells were transfected into the negative control RNA (NC) or miR-145 precursor (miR-145) and seeded in 6-well plates. After 2 weeks, the number of colonies was counted, and colony-forming efficiency is shown ( $\left.{ }^{*} \mathrm{P}<0.05\right)$. (C) Cell cycle analysis was performed by flow cytometry (FCM), and the percentages of SMMC-7721 cells in the $\mathrm{G} 1, \mathrm{G} 2$ and $\mathrm{S}$ phase are shown (all, $\mathrm{P}>0.05$ ).

A

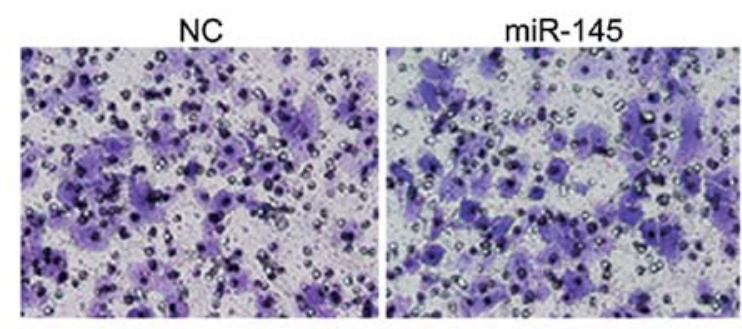

B

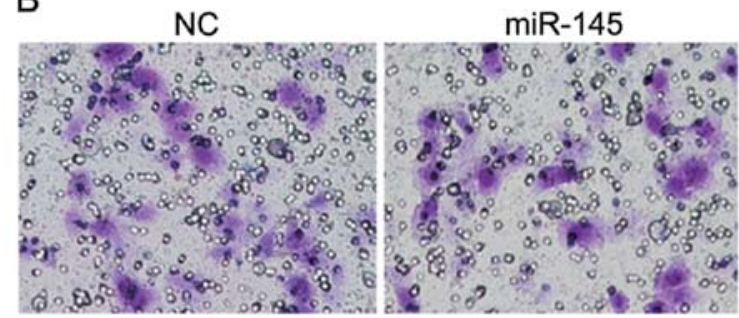



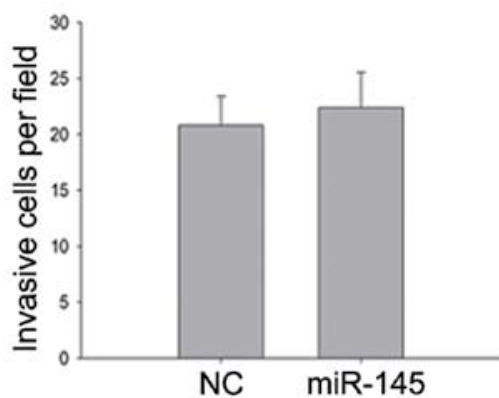

Figure 4. miR-145 affects the migration and invasion of SMMC-7721 cells. (A) Transwell migration assays were carried out and the results show that miR-145 overexpression does not affect the cell migration ability of SMMC-7721 cells $(\mathrm{P}=0.886)$. (B) Transwell invasion assays were carried out and that miR-145 overexpression does not affect cell invasion ability of SMMC-7721 cells $(\mathrm{P}=0.404)$.

slightly increase in the number of cells in the G2 phase of the cell cycle, although this result was not statistically significant $(\mathrm{P}=0.693$, the two-tailed Student's t-test). Similarly, there was no significant difference for the amount of G1-phase $(\mathrm{P}=0.798$, the two-tailed Student's t-test) and S-phase ( $\mathrm{P}=0.877$, the twotailed Student's t-test) (Fig. 3C).
miR-145 does not affect cell migration and invasion ability. Migration and invasion ability of SMMC-7721 cells transfected with miR-145 precursor or negative control were determined by Matrigel chamber assays. The results demonstrated that the overexpression of miR-145 did not exert any significant effect on the migration $(\mathrm{P}=0.886$, the two-tailed 
A

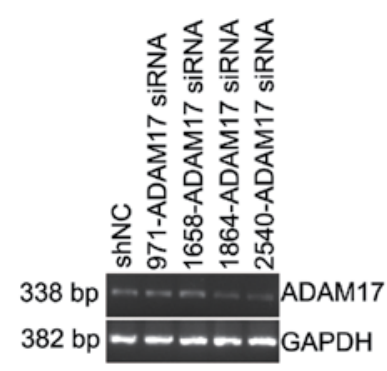

C

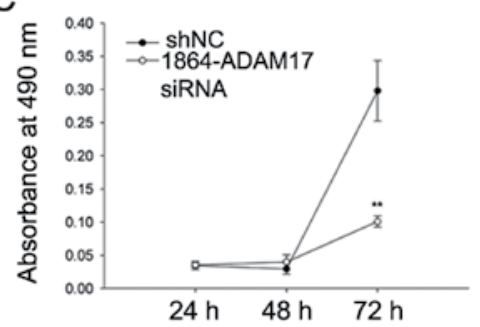

B

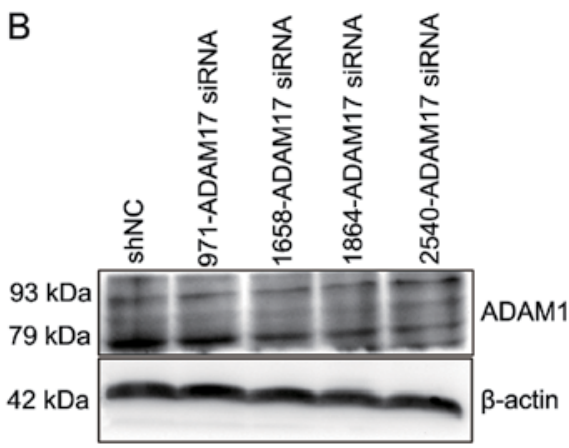

D

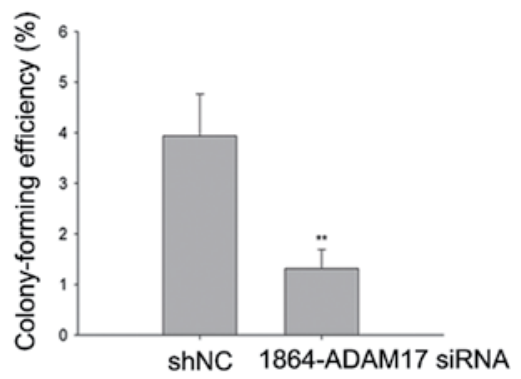

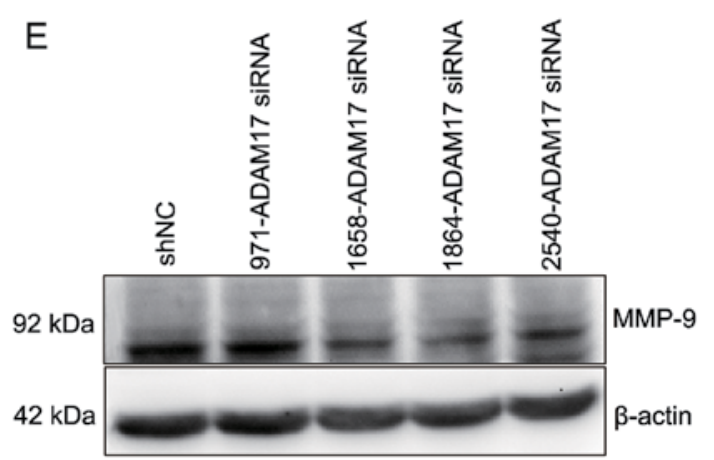

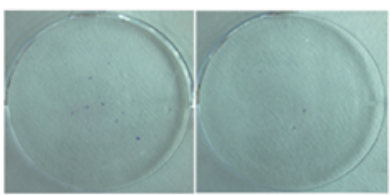

Figure 5. Silencing of ADAM17 suppresses cell growth and colony formation and decreases the expression of MMP-9. (A and B) SMMC-7721 cells were infected with four different ADAM17 siRNAs or negative control shRNA. ADAM17 mRNA and protein expression levels were markedly decreased in SMMC-7721 cell transfected with ADAM17 siRNA as compared to the negative control shRNA (shNC). (C) Proliferation of SMMC-7721 cells at 72 h after ADAM17 siRNA (1864-ADAM17 siRNA) transfection was significantly decreased as compared to shNC $\left({ }^{* *} \mathrm{P}<0.01\right)$. (D) The colony formation assay was performed on the SMMC-7721 cells transfected with ADAM17 siRNA or shNC, and the colony-forming efficiency was decreased by ADAM17 knockdown (** $<0.01)$. (E) MMP-9 expression was markedly decreased in SMMC-7721 cells transfected with ADAM17 siRNA, as compared to shNC. ADAM17, A disintegrin and metalloproteinase 17.

Student's t-test) (Fig. 4A) and invasion ability $(\mathrm{P}=0.404$, the two-tailed Student's t-test) (Fig. 4B) of SMMC-7721 cell lines.

Silencing of ADAM17 inhibited the growth of cells and reduced the expression of MMP-9 in SMMC-7721 cells. To clarify the mechanisms by which ADAM17 decreases cell proliferation, we suppressed ADAM17 expression using siRNA. RT-PCR and western blot analysis showed that the expression levels of ADAM17 mRNA and protein were significantly lower (Fig. 5A and B). The MTT assay showed that this suppression of ADAM17 markedly reduced the proliferation ability of SMMC-7721 cells relative to the control cells at $72 \mathrm{~h}(\mathrm{P}<0.01$, Mann-Whitney $U$ test), however it did not exhibit any change at $24 \mathrm{~h}(\mathrm{P}=0.762$, the two-tailed Student's t-test $)$ and $48 \mathrm{~h}(\mathrm{P}=0.083$, the two-tailed Student's t-test) (Fig. 5C). The colony-forming test assay demonstrated that the overexpression of miR-145 markedly inhibited cell proliferation in SMMC-7721 cells $(\mathrm{P}<0.05$, Mann-Whitney U test) (Fig. 5D). At the same time, ADAM17
siRNA caused th edownregulation of MMP-9 (Fig. 5E). It has been reported that MMP-9 proteolytically activates TGF- $\beta$ and promotes tumor growth and invasion (31). Therefore, these results suggested that suppression of cell growth may be connected to ADAM17 siRNA reducing the expression of MMP-9.

\section{Discussion}

Accumulating evidence suggests a role for microRNAs (miRNAs) whereby they regulate cancer development and progression (32-34). It is of vital importance to understand the functions and mechanisms of cancer-specific miRNAs for cancer therapy and prevention. In the present study, we found that ADAM17 is a target gene of miR-145. The experimental results showed that the overexpression of miR-145 led to a marked reduction of ADAM17 expression in different HCC cells. Moreover, the ability of miR-145 to downregulate ADAM17 expression was performed through 
a drect combination with the complimentary sequence in the 3'UTR of ADAM17 mRNA to the miR-145. Notably, miR-145 significantly inhibited the growth activity of SMMC-7721 cells. These findings suggest that miR-145 plays a tumor-suppressor role in hepatocarcinogenesis by targeting ADAM17.

ADAM17 is one of the members of the family of ADAMs (a disintegrin and metalloproteinase). Previous studies have confirmed that ADAM17 is important in cancer formation and progression (35-37). Notably, apart from miR-145, various miRNA molecules can regulate the expression of ADAM17. For example, miR-26 mediated the observed effects on white and brite adipogenesis by targeting ADAM17 (38). Chen et al reported that hydroquinone-induced miR-122 downregulation resulted in the upregulation of ADAM17 expression in human leukemic cells (39). miR-122 affects intrahepatic metastasis in HCC by regulating the expression of ADAM17 (40). Thus, the downregulation of ADAM17 is important in miR-145-mediated suppression of cell growth and metastasis. In the present study, the ADAM17 siRNA can reduce the protein expression of ADAM17 in SMMC-7721 cells and suppress the growth and proliferation activity of SMMC-7721 cells, which serves as evidence for our previous hypothesis.

The molecular mechanism of ADAM17 has been shown to be responsible for the growth inhibition of cancer cells. Das et al reported that ADAM17 silencing decreased the expression of vascular endothelial growth factor-A (VEGF-A) and MMP-9 in murine colon carcinoma cells (MC38CEA) and exhibited its antitumor immune response (41). Xiao et al found that the knockdown of ADAM17 can deactivate the EGFR-MEK-ERK signaling pathway, resulting in the downregulation of MMP-2 and MMP-9, therby reducing the invasive ability of prostate cancer cells (42). ADAM17 siRNA may suppress cancer growth by decreasing the expression of MMP-9. MMP-9 is expressed as a $92-\mathrm{kDa}$ proenzyme and is converted to the active $83-\mathrm{kDa}$ mature enzyme (43). MMP-9 plays a significant role in different stages of tumor progression and metastasis, and its inhibitory role in cell signaling pathways can block MMP-9-mediated cell migration and metastasis (44-46). Results of the present study have shown that the silencing of ADAM17 reduces the expression of MMP-9 in SMMC-7721 cells. Therefore, we suggest that there is likely a link between the two proteins in tumorigenesis. In addition, it is possible that the miR-145-mediated suppression of cell proliferation was performed through the ADAM17/ MMP-9 pathway.

As a promising miRNA, the therapeutic potential of miR-145 is important in examining the relationship between miR-145 and other mRNAs in hepatocarcinogenesis. The present study has demonstrated that miR-145 suppressed the proliferation of HCC cells by targeting ADAM17, suggesting miR-145 has the potential to be applied in tumor treatment. This result led to investigatation of the anti-neoplastic effect of miR-145. In general, the results suggest that miR-145 may be crucial in miRNA-base anticancer therapies in the future.

\section{Acknowledgements}

We would like to thank Dr Zhaojun Duan for her excellent technical assistance. This study was supported by the National Natural Science Foundation of China (no. 81270501), and the Exploring Program of Central South University (2012QNZT080).

\section{References}

1. Bartel DP: MicroRNAs: genomics, biogenesis, mechanism, and function. Cell 116: 281-297, 2004.

2. Calin GA and Croce CM: MicroRNA signatures in human cancers. Nat Rev Cancer 6: 857-866, 2006.

3. Kloosterman WP and Plasterk RH: The diverse functions of microRNAs in animal development and disease. Dev Cell 11: 441-450, 2006.

4. Kim VN and Nam JW: Genomics of microRNA. Trends Genet 22: 165-173, 2006.

5. Lewis BP, Burge CB and Bartel DP: Conserved seed pairing, often flanked by adenosines, indicates that thousands of human genes are microRNA targets. Cell 120: 15-20, 2005.

6. Kerr TA, Korenblat KM and Davidson NO: MicroRNAs and liver disease. Transl Res 157: 241-252, 2011.

7. Siegel R, Naishadham D and Jemal A: Cancer statistics, 2012. CA Cancer J Clin 62: 10-29, 2012.

8. Takahashi K, Yan I, Wen HJ and Patel T: microRNAs in liver disease: from diagnostics to therapeutics. Clin Biochem 46: 946-952, 2013.

9. Sachdeva M and Mo YY: miR-145-mediated suppression of cell growth, invasion and metastasis. Am J Transl Res 2: 170-180, 2010.

10. Zhang B, Pan X, Cobb GP and Anderson TA: microRNAs as oncogenes and tumor suppressors. Dev Biol 302: 1-12, 2007.

11. Zhao C, Xu Y, Zhang Y, et al: Downregulation of miR-145 contributes to lung adenocarcinoma cell growth to form brain metastases. Oncol Rep 30: 2027-2034, 2013.

12. Havelange V, Stauffer N, Heaphy CC, et al: Functional implications of microRNAs in acute myeloid leukemia by integrating microRNA and messenger RNA expression profiling. Cancer 117: 4696-4706, 2011.

13. Sachdeva M and Mo YY: MicroRNA-145 suppresses cell invasion and metastasis by directly targeting mucin 1 . Cancer Res 70: 378-387, 2010.

14. Gregersen LH, Jacobsen AB, Frankel LB, Wen J, Krogh A and Lund AH: MicroRNA-145 targets YES and STAT1 in colon cancer cells. PLoS One 5: e8836, 2010.

15. Noh JH, Chang YG, Kim MG, et al: MiR-145 functions as a tumor suppressor by directly targeting histone deacetylase 2 in liver cancer. Cancer Lett 335: 455-462, 2013.

16. Cho WC, Chow AS and Au JS: MiR-145 inhibits cell proliferation of human lung adenocarcinoma by targeting EGFR and NUDT1. RNA Biol 8: 125-131, 2011.

17. Lee HK, Bier A and Cazacu S, et al: MicroRNA-145 is downregulated in glial tumors and regulates glioma cell migration by targeting connective tissue growth factor. PLoS One 8: e54652, 2013.

18. Doberstein K, Steinmeyer N, Hartmetz AK, et al: MicroRNA-145 targets the metalloprotease ADAM17 and is suppressed in renal cell carcinoma patients. Neoplasia 15: 218-230, 2013.

19. Lu Y, Chopp M, Zheng X, Katakowski M, Buller B and Jiang F: MiR-145 reduces ADAM17 expression and inhibits in vitro migration and invasion of glioma cells. Oncol Rep 29: 67-72, 2013.

20. Yang XW, Zhang LJ, Huang XH, et al: miR-145 suppresses cell invasion in hepatocellular carcinoma cells: miR-145 targets ADAM17. Hepatol Res 44: 551-559, 2014.

21. Black RA, Rauch CT, Kozlosky CJ, et al: A metalloproteinase disintegrin that releases tumour-necrosis factor- $\alpha$ from cells. Nature 385: 729-733, 1997.

22. Mohan MJ, Seaton T and Mitchell J, et al: The tumor necrosis factor- $\alpha$ converting enzyme (TACE): a unique metalloproteinase with highly defined substrate selectivity. Biochemistry 41: 9462-9469, 2002.

23. Zheng X, Jiang F, Katakowski M, Zhang ZG, Lu QE and Chopp M: ADAM17 promotes breast cancer cell malignant phenotype through EGFR-PI3K-AKT activation. Cancer Biol Ther 8: 1045-1054, 2009.

24. Zheng X, Jiang F, Katakowski M, Lu Y and Chopp M: ADAM17 promotes glioma cell malignant phenotype. Mol Carcinog 51: $150-164,2012$.

25. Lee SO, Jeong YJ, Yu MH, et al: Wogonin suppresses TNF- $\alpha$-induced MMP-9 expression by blocking the NF- $\mathrm{kB}$ activation via MAPK signaling pathways in human aortic smooth muscle cells. Biochem Biophys Res Commun 351: 118-125, 2006. 
26. Balasubramanian S, Fan M, Messmer-Blust AF, et al: The interferon- $\gamma$-induced GTPase, mGBP-2, inhibits tumor necrosis factor $\alpha(\mathrm{TNF}-\alpha)$ induction of matrix metalloproteinase- 9 (MMP-9) by inhibiting NF- $\mathrm{BB}$ and Rac protein. J Biol Chem 286: 20054-20064, 2011.

27. Li YQ, Yan JP, Xu WL, et al: ADAM17 mediates MMP9 expression in lung epithelial cells. PLoS One 8: e51701, 2013.

28. Elbashir SM, Harborth J, Weber K and Tuschl T: Analysis of gene function in somatic mammalian cells using small interfering RNAs. Methods 26: 199-213, 2002.

29. Krek A, Grün D, Poy MN, et al: Combinatorial microRNA target predictions. Nat Genet 37: 495-500, 2005.

30. John B, Enright AJ, Aravin A, Tuschl T, Sander C and Marks DS Human microRNA targets. PLoS Biol 2: e363, 2004.

31. Yu Q and Stamenkovic I: Cell surface-localized matrix metalloproteinase- 9 proteolytically activates TGF- $\beta$ and promotes tumor invasion and angiogenesis. Genes Dev 14: 163-176, 2000.

32. Ma L, Teruya-Feldstein J and Weinberg RA: Tumour invasion and metastasis initiated by microRNA-10b in breast cancer Nature 449: 682-688, 2007

33. Zhu S, Wu H, Wu F, Nie D, Sheng S and Mo YY: MicroRNA-21 targets tumor suppressor genes in invasion and metastasis. Cell Res 18: 350-359, 2008.

34. Kusenda B, Mraz M, Mayer J and Pospisilova S: MicroRNA biogenesis, functionality and cancer relevance. Biomed Pap Med Fac Univ Palacky Olomouc Czech Repub 150: 205-215, 2006.

35. McGowan PM, Ryan BM, Hill AD, McDermott E, O'Higgins N and Duffy MJ: ADAM-17 expression in breast cancer correlates with variables of tumor progression. Clin Cancer Res 13: 2335-2343, 2007.

36. Kenny PA and Bissell MJ: Targeting TACE-dependent EGFR ligand shedding in breast cancer. J Clin Invest 117: 337-345, 2007.
37. Borrell-Pagès $\mathrm{M}$, Rojo $\mathrm{F}$, Albanell $\mathrm{J}$, Baselga $\mathrm{J}$ and Arribas $\mathrm{J}$ : TACE is required for the activation of the EGFR by TGF- $\alpha$ in tumors. EMBO J 22: 1114-1124, 2003.

38. Karbiener M, Pisani DF, Frontini A, et al: MicroRNA-26 family is required for human adipogenesis and drives characteristics of brown adipocytes. Stem Cells 32: 1578-1590, 2014.

39. Chen YJ and Chang LS: Hydroquinone-induced miR-122 downregulation elicits ADAM17 up-regulation, leading to increased soluble TNF- $\alpha$ production in human leukemia cells with expressed Bcr/Abl. Biochem Pharmacol 86: 620-631, 2013.

40. Tsai WC, Hsu PW, Lai TC, et al: MicroRNA-122, a tumor suppressor microRNA that regulates intrahepatic metastasis of hepatocellular carcinoma. Hepatology 49: 1571-1582, 2009.

41. Das S, Czarnek M, Bzowska M, et al: ADAM17 silencing in mouse colon carcinoma cells: the effect on tumoricidal cytokines and angiogenesis. PLoS One 7: e50791, 2012.

42. Xiao LJ,Lin P, Lin F, et al: ADAM17 targets MMP-2 and MMP-9 via EGFR-MEK-ERK pathway activation to promote prostate cancer cell invasion. Int J Oncol 40: 1714-1724, 2012.

43. Mattu TS, Royle L, Langridge J, et al: O-glycan analysis of natural human neutrophil gelatinase $\mathrm{B}$ using a combination of normal phase-HPLC and online tandem mass spectrometry: implications for the domain organization of the enzyme. Biochemistry 39: 15695-15704, 2000.

44. Kessenbrock K, Plaks V and Werb Z: Matrix metalloproteinases: regulators of the tumor microenvironment. Cell 141: 52-67, 2010.

45. Bauvois B: New facets of matrix metalloproteinases MMP-2 and MMP-9 as cell surface transducers: outside-in signaling and relationship to tumor progression. Biochim Biophys Acta 1825: 29-36, 2012.

46. Klein T and Bischoff R: Physiology and pathophysiology of matrix metalloproteases. Amino Acids 41: 271-290, 2011. 